\title{
Characterizing Quotation
}

\author{
Chung-chieh Shan \\ Rutgers University
}

\section{Introduction}

Davidson (1979) distinguished mixed quotation, as in (1) and (2), from pure, direct, and indirect quotation, as in (3)-(5).

(1) (Mixed quotation) Quine says quotation 'has a certain anomalous feature'.

(2) (Mixed quotation) Bush is proud of his 'eckullectic' reading list.

(3) (Pure quotation) 'Bachelor' has eight letters.

(4) (Direct quotation) Quine says 'quotation has a certain anomalous feature'.

(5) (Indirect quotation) Quine says quotation has a certain anomalous feature.

The quotes in (3) and (4) mention the quoted expression without using them. We can analyze these quotes as simply denoting the quoted expressions, which have properties such as having eight letters and being said by Quine. In contrast, the embedded clause in (5) is a quote only in that it uses - not mentions - the expression quotation has a certain anomalous feature to express a proposition attributed to Quine. Hence, it is easy to imagine a situation where (5) holds but (4) does not: perhaps Quine never uttered the word anomalous.

What is special about the quotes in (1) and (2) is that they mix mention and use. On one hand, these sentences hardly hold if Quine never uttered anomalous and Bush never uttered eckullectic. On the other hand, in order to compose the meanings of quotation 'has a certain anomalous feature' and 'eckullectic' reading list, we need the quotes to denote verb-phrase and adjectival meanings, which can apply to quotation and reading lists, rather than expressions, which cannot.

One way to analyze the syntax and semantics of mixed quotation is to formulate some syntactic and semantic rules that generate a fragment of English with mixed quotation. This paper presents such a formal fragment, to show that quotation has a recursive and compositional structure, as we have come to expect in language and study in linguistics. It turns out in the fragment that quoted expressions denote characters (Kaplan 1989), so the semantics of quotation simulates the pragmatics of speech, including dependence on utterance contexts and reference to mental entities. The analysis also accommodates varieties of unquotation, pure quotation, and causal reference (Putnam 1975, Kripke 1980).

Thanks to Chris Barker, Ivano Caponigro, Sam Cumming, David Dowty, Gabriel Greenberg, Michael Johnson, Ezra Keshet, Dan Lassiter, Ernie Lepore, Emar Maier, Matthew Stone, Rutgers linguistics, University of Århus, Radboud University Nijmegen, and the SALT XIX organizers and audience. 


\subsection{The Truth Conditions of Mixed Quotation}

Before writing a fragment, we need to clarify the goal: what are the truth conditions and well-formedness conditions of mixed quotation? As the term mixed suggests (Geurts and Maier 2003, Potts 2007), the sentences (1) and (2) have two dimensions of meaning, one of mention and one of use. I gloss them separately below:

Quine says quotation 'has a certain anomalous feature'.

a. (mention) has a certain anomalous feature is used to mean some $f$.

b. (use) Quine says quotation $f$.

(7) Bush is proud of his 'eckullectic' reading list.

a. (mention) eckullectic is used to mean some $f$.

b. (use) Bush is proud of his $f$ (reading list).

In each example, the use dimension depends anaphorically on the mention dimension for a semantic value $f$, which is a verb-phrase or adjectival meaning.

The phrase 'is used to mean' above skirts two difficult issues. First, although the most obvious readings by far of the sentences (1) and (2) require Quine and Bush to use the quoted expressions, the truth conditions in (6) and (7) only require someone to. This weakness is actually a strength, because not every quoted expression has its speaker even mentioned in the quoting sentence. For example, the most natural way to interpret the continuation (8) is that 'eckullectic' quotes Bush rather than Cheney.

(8) Yet Cheney's reading list is far more 'eckullectic', not to mention longer.

In general, an embedding context such as Quine says or Bush is proud of is just a clue that helps the hearers of a mixed quote resolve who is being quoted, just as they disambiguate among multiple grammatical parses of a string. The quote may even be hypothetical or generic. It is thus natural to analyze the mention dimension of a mixed-quote meaning as a presupposition, as Geurts and Maier (2003) do, or as a conventional implicature, as Potts (2007) does. In contrast, the use dimension of a mixed-quote meaning is the semantic value $f$ it contributes to the sentence's at-issue content (Potts 2003). Following these authors, I content myself with a fragment that 'bakes in' the result of the resolution and just generates sentences containing mixed quotes of Quine and Bush with truth conditions such as (6b) and (7b).

The second issue is, what does it take for someone to use some expression to mean something? When Quine (1940) wrote

(9) it has a certain anomalous feature which calls for special caution

he not only used that clause to mean a proposition, but also used various parts of that clause to mean various semantic values. It was by virtue of these values and their composition that the clause came to mean the proposition. ${ }^{1}$ In this way,

\footnotetext{
${ }^{1}$ That is not to say the parts form a hierarchy: the overlapping parts has a certain, has a certain anomalous feature, and a certain anomalous feature which calls for special caution were each used by Quine to mean something yet do not contain each other. Barker (2007) describes how to identify the semantic value contributed by each part without nailing down a single hierarchy of composition.
} 
an utterance context - that is, an utterance event or occasion, be it hypothetical or generic or not-comprises numerous utterance subevents in a network that mirrors the syntactic structure of the utterance. In each subevent, some expression was used to mean something. Hence, (7) may be true even if eckullectic is heretofore used to describe shoes only.

One intuition behind this gloss of mixed quotation is that using an expression to mean a value is like decoding a Gödel number to yield a proposition or running a program to compute a result. Each of these operations is a systematic mapping from forms to meanings in an object language. In the case of mixed quotation, the object language is not mathematical logic or computer code but Quine English or Bush English. The quoted speaker's mind provides a mapping from public, spoken forms to private, intended meanings, so mixed quotation lets us curate meanings from other minds into our own utterances. As the fragment below shows, we can regard our uncertainty about what others mean as a special case of uncertainty about the world. For example, without knowing which 'anomalous feature' Quine had in mind or what it takes for Bush to consider something 'eckullectic', we can refer to these values by mixed-quoting Quine and Bush. Similarly, we can appeal to a botanist's notion of an elm and a historian's notion of Aristotle by mixed-quoting them (Putnam 1975, Kripke 1980), if without punctuating the written English by quotation marks.

Of course, mixed quotation often serves more purpose in discourse than referring to what an expression is used to mean. For example, a mixed quote often signals that the quoting speaker is distant from the quoted speaker and would not use the quoted expression in the same way. I focus here on the truth and wellformedness conditions of mixed quotation, leaving it to future work to explain the other accomplishments of a mixed quote as different ways to use it (Cappelen and Lepore 2003).

\subsection{The Well-Formedness Conditions of Mixed Quotation}

The mixed quotes in (1) and (2) function grammatically as a verb phrase and an adjective respectively. Not all mixed quotes can, as the following attempts illustrate:

(10) * Bush said his reading list 'eckullectic'.

(11) * Quine's 'has a certain anomalous feature' is trivial.

The unacceptability of (10) demonstrates that a mixed quote of an adjective in Bush English cannot serve as a verb phrase. Perhaps this case is just a semantic type mismatch - after all, in the standard analysis, a verb phrase denotes a property whereas an adjective denotes a function from properties to properties. But it is also standard for a common noun to denote a property, so a semantic type mismatch does not account for the unacceptability of (11), in which a mixed quote of a verb phrase in Quine English fails to serve as a common noun. What does account for the unacceptability of (10) and (11) is that the syntactic category of a mixed quote must match (to a first approximation, equal) that of the quoted expression. 
In fact, this requirement for the categories to match extends from the coarse distinction between verb phrases and common nouns to finer agreement in number and gender. For example, suppose Ken has said

(12) My only paper has appeared in print.

It would be either false or unacceptable for someone to report

(13) Ken has said literally that all of his papers ' $\{$ have/*has $\}$ appeared in print'.

Similarly for gender, suppose Ken has said in Italian

Gli uomini italiani mi sembrano molto carini

the.MASC.PL men Italian.MASC.PL to.me look.3PL very cute.MASC.PL

'Italian men look very cute to me.'

It would be either false or unacceptable for someone to report

Ken ha detto letteralmente che le persone italiane mi

Ken has said literally that the.FEM.PL people Italian.FEM.PL to.me sembrano molto carine/*carini'

look.3PL very cute.FEM.PL/*cute.MASC.PL

'Ken has said literally that Italian people 'mi sembrano molto carine/carini'.'

Perhaps the mixed quotes in (10) and (11) wrongly presuppose that eckullectic is used as a verb phrase and has a certain anomalous feature is used as a common noun. On such an account, (10) and (11) are bad the same way (16) is: due to presupposition failure.

* Bush met the king of France.

Seeing as the constraint at work is effectively a relation between the syntactic categories of the quoting and quoted languages, I explore in this paper how to retrieve it from the pragmatic wastebasket and express it by syntactic means, if only to avoid duplicating machinery. In other words, I treat (10) and (11) as syntactically ill-formed, on a par with the following counterparts without mixed quotation.

(17) * Bush said his reading list eclectic.

(18) * Quine's constitutes a knockdown argument is trivial.

\section{The Basic Fragment: Embedding Languages}

We are ready for a fragment that formally expresses the truth and well-formedness conditions just set forth. The main idea is for our syntactic categories to embed those of the quoted languages and for our semantic values to include the characters of the quoted languages. 
We start with a standard categorial grammar with an intensional (possibleworlds) semantics. In this language, eclectic is an adjective, but not eckullectic.

$$
\begin{aligned}
& A::=A / B \quad B \\
& \llbracket A \rrbracket(w)=\llbracket A / B \rrbracket(w)(\llbracket B \rrbracket) \\
& A::=B \quad B \backslash A \\
& \llbracket A \rrbracket(w)=\llbracket B \backslash A \rrbracket(w)(\llbracket B \rrbracket) \\
& \text { DP }::=\text { Bush } \\
& (\mathrm{DP} \backslash \mathrm{S}) / \mathrm{S}::=\text { says } \\
& (\mathrm{DP} \backslash \mathrm{S}) / \mathrm{DP}::=\text { is proud of } \\
& \mathrm{N} / \mathrm{N}::=\text { eclectic } \\
& \mathrm{N}::=\text { reading list }
\end{aligned}
$$

For each syntactic category $A$, constituents of category $A$ have semantic contents of type $\langle s, \tau(A)\rangle$, where $s$ is the type of worlds and $\tau(A)$ is defined as usual by

$$
\tau(A / B)=\tau(B \backslash A)=\langle\langle s, \tau(B)\rangle, \tau(A)\rangle, \quad \tau(\mathrm{DP})=e, \quad \tau(\mathrm{S})=t, \quad \ldots
$$

I write $\llbracket \cdot \rrbracket$ for the content of an expression · in our language. Starting in the semantic rules to the right of (19) and (20), I abuse notation and write $\llbracket A \rrbracket$ to mean the content of the constituent of category $A$.

In preparation for mixed quotation, for each category $A$ of Bush English (the quoted language), we add a category $A^{\prime}$ to our language (the quoting language).

$$
\begin{aligned}
(\mathrm{N} / \mathrm{N})^{\prime}: & :=\text { e eckullectic } & \llbracket(\mathrm{N} / \mathrm{N})^{\prime} \rrbracket(w)(i) & =\llbracket \text { eckullectic } \rrbracket_{w}^{i} \\
\mathrm{~N}^{\prime}: & :=\text { reading list } & \llbracket \mathrm{N}^{\prime} \rrbracket(w)(i) & =\llbracket \text { reading list } \rrbracket_{w}^{i}
\end{aligned}
$$

In the syntax of our quoting language, these primed categories $A^{\prime}$ embed the syntax of a quoted language. For example, our categories $(\mathrm{N} / \mathrm{N})^{\prime}$ and $\mathrm{N}^{\prime}$ house adjectives and common nouns in Bush English. We keep these categories distinct from $\mathrm{N} / \mathrm{N}$ and $\mathrm{N}$, to exclude Bushisms such as

\section{* Bush is proud of his eckullectic reading list}

from our category S. This prime is reserved for Bush-to quote Quine as well, we would need another family of primed categories, perhaps $A^{\prime}$. Similarly, to deal with nested quotes as in (30), where the quoted language includes quotes itself, we would need doubly primed categories, but the basic account is the same.

The politician said she is "sorry to have used an "epithet".

As for semantics, I write $\llbracket \cdot \rrbracket_{w}^{i}$ for the content that an expression $\cdot$ is used to mean in an utterance context $i$ in a world $w .^{2}$ For example, assuming that Bush uses eckullectic to mean 'eclectic' and reading list to mean simply 'reading list' in some context $i$ in the real world $w_{0}$, we have

$$
\llbracket \text { eckullectic } \rrbracket_{w_{0}}^{i}=\llbracket \text { eclectic } \rrbracket \quad \text { and } \quad \llbracket \text { reading list } \rrbracket_{w_{0}}^{i}=\llbracket \text { reading list } \rrbracket .
$$

\footnotetext{
${ }^{2}$ Perhaps each context belongs to a unique world. In that case, $w_{1}=w_{2}$ whenever $\llbracket \cdot \rrbracket_{w_{1}}^{i}$ and $\llbracket \cdot \rrbracket_{w_{2}}^{i}$ are both defined.
} 
As the argument $i$ in (27) and (28) indicates, a quoting expression in our language denotes the character (Kaplan 1989) - a function from utterance contexts to semantic contents - of the quoted expression. Accordingly, we define $\tau\left(A^{\prime}\right)=$ $\langle u,\langle s, \tau(A)\rangle\rangle$, where $u$ is the type of utterance contexts. In short, quoting categories embed quoted categories, and quoting denotations are quoted characters. We next put this correspondence to work.

So far, our language is merely a disjoint union of normal and Bush English. To pass between the two, we add a rule for mixed quotation.

$$
A::=A^{\prime}, \quad \llbracket A \rrbracket(w)=\llbracket A^{\prime} \rrbracket(w)(\text { Bush English }(\text { in } w))(w)
$$

In syntax, for any category $A$, putting quotation marks around (or quotation intonation on) an $A$-expression in Bush English makes it an $A$-expression in our language. For example, both eclectic and 'eckullectic' are in our category N/N but not DP $\backslash \mathrm{S}$, so our fragment generates sentences such as (2) alongside the ordinary

(33) Bush is proud of his eclectic reading list

but not (10) or (17). For comparison, the derivation trees are as follows.

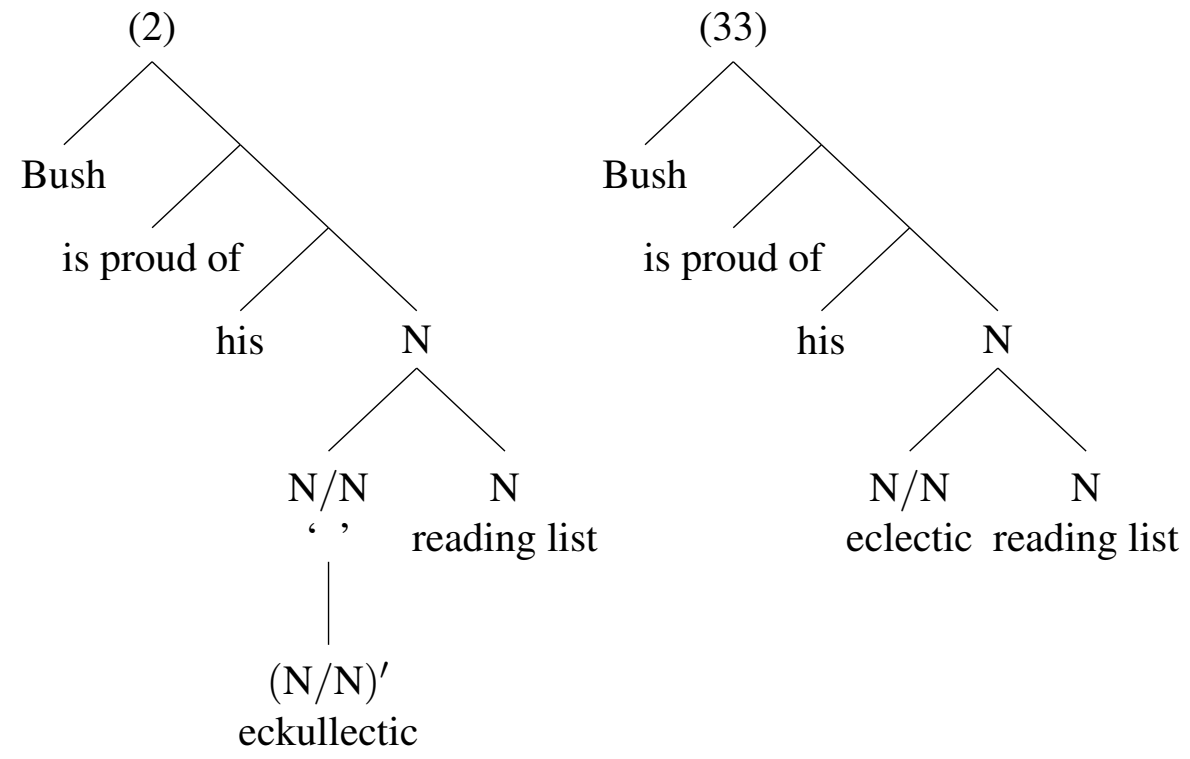

The semantic rule in (32) specifies a form of diagonalization, which Stalnaker (1978) originally proposed as a pragmatic operation. Specifically, the first two arguments ${ }^{3}$ passed to $\llbracket A^{\prime} \rrbracket$ compute what Bush uses the quoted expression to mean in

\footnotetext{
${ }^{3}$ I am not committed to this use of two arguments, a possible world and an utterance context, to distinguish among the different semantic contents that a given quoted expression such as eckullectic may be used to mean. If each context belongs to a unique world-in particular, if we 'represent contexts by the same indexed sets we use to represent circumstances' as Kaplan (1989) put it — then these two arguments can be merged into one, namely Bush English in $w$, as a matter of presentation. In other words, I am not committed as to whether it is really quoting denotations or quoting senses that are quoted characters.
} 
the world $w$, then the last argument $w$ extracts from this semantic content its denotation at $w$. The second argument 'Bush English (in $w$ )' stands for some utterance context, perhaps a generic one, in which Bush uses the quoted expression.

Let us put these rules together to generate some simple examples. On one hand, if we know that Bush means 'eclectic' by eckullectic - in other words, if

$$
\llbracket \text { eckullectic } \rrbracket_{w}^{\text {Bush English }}=\llbracket \text { eclectic } \rrbracket
$$

for every belief world $w$ of ours - then (2) is grammatical and true in exactly those worlds where Bush is proud of his eclectic reading list, as desired. On the other hand, if we do not know the semantics of the quoted language so well, then sentences containing mixed quotes can inform us about it. If $\llbracket$ eckullectic $\rrbracket_{w}^{\text {Bush English }}$ is not constant across our belief worlds $w$, then the two sentences below are not synonymous: a world satisfies (36) if and only if to be what Bush means by eckullectic in $w$, be it 'eclectic' or not, is to have never been seen by Bush.

To be 'eckullectic' is to have never been seen by Bush.

\section{To be eclectic is to have never been seen by Bush.}

This interpretation strategy is essentially how Stalnaker (1978) dealt with sentences like (38): by treating Hesperus and Phosphorus as mixed quotes of normal English.

Hesperus is identical with Phosphorus.

One reason to let quoting denotations be characters, and to locate the diagonalization operation of mixed quotation in semantics rather than pragmatics, is that mixed quotation allows quantifying over utterance contexts. For example, the most salient reading of the sentence

Every day, I would promise to finish the paper 'tomorrow'

refers to not a single context of uttering tomorrow but one per day. Given that the character of tomorrow maps different contexts to different times, we can account for this reading by resolving (in the sense of Section 1.1) the context of tomorrow so that it depends on and covaries with the day. To take another example, the sentence

Danes and Norwegians eat 'frokost' at different times

has one reading that is true because frokost means 'lunch' in Danish and 'breakfast' in Norwegian. We can account for this reading by resolving the context of frokost so that it depends on and covaries with the subject.

\section{The Productivity of Quotation and Unquotation}

Given that our fragment already contains individual expressions of Bush English, it is a small step to also add the combination modes of Bush English. Assuming 
that Bush English allows forward and backward application as usual, we add corresponding primed rules:

$$
\begin{array}{ll}
A^{\prime}::=(A / B)^{\prime} B^{\prime} & \llbracket A^{\prime} \rrbracket(w)(i)=\llbracket A::=A / B B \rrbracket_{w}^{i}\left(\llbracket(A / B)^{\prime} \rrbracket(w)(i), \llbracket B^{\prime} \rrbracket(w)(i)\right) \\
A^{\prime}::=B^{\prime}(B \backslash A)^{\prime} & \llbracket A^{\prime} \rrbracket(w)(i)=\llbracket A::=B B \backslash A \rrbracket_{w}^{i}\left(\llbracket B^{\prime} \rrbracket(w)(i), \llbracket(B \backslash A)^{\prime} \rrbracket(w)(i)\right)
\end{array}
$$

You never know if the semantics of Bush English handles forward and backward application in the same way as normal English (namely (19) and (20)). Hence, we let the composition functions vary: In the semantic rules above, $\llbracket A:=A / B B \rrbracket_{w}^{i}$ stands for what forward application is used to mean in an context $i$ in a world $w$. It is a function that maps an ordered pair of semantic contents, of quoted $(A / B)$ - and $B$-expressions, to the semantic content of the combined quoted $A$-expression. The upshot is that our semantic composition refers to and simulates Bush's.

A first payoff of these primed rules is that they parsimoniously account for the productivity of quotes in generic contexts. A simple example of this productivity is that code switching can be regarded as mixed-quoting a generic context, such as that of French utterances, and is not restricted to a finite set of expressions, such as those that have been uttered before. To take a less mundane example, suppose that Bush is predisposed to use eckullectic to mean 'eclectic' and reading list to mean 'prepared speech', not just occasionally but regularly and habitually. Even if he never uses both phrases in the same actual utterance, then, Bush uses eckullectic to mean 'eclectic' and reading list to mean 'prepared speech' in a single generic context. The sentence

\section{Bush is proud of his 'eckullectic reading list'}

may thus be acceptable and true in that Bush is proud of his eclectic prepared speech. Without any explicit production such as ' $\mathrm{N}^{\prime}::=$ eckullectic reading list', our fragment generates this sentence by combining (27) and (28) using (41) and (32):

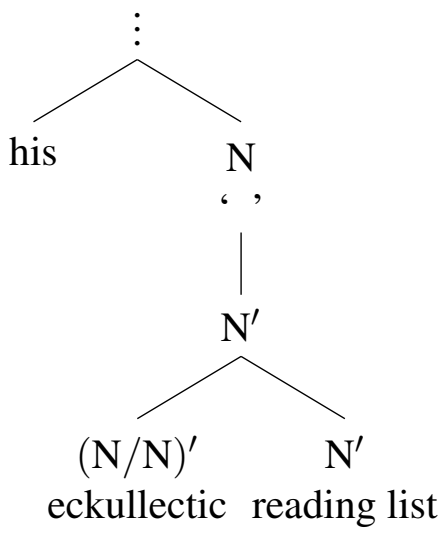

A more significant payoff of the primed rules is that they let us account for unquotation: including non-quoted material inside a quote (Bawden 1999). In written English, unquotation is typically punctuated using square brackets. However, square brackets are ambiguous between syntactic and semantic unquotation (Shan 
2007), as the following examples of mixed quotation illustrate.

(45) (Syntactic unquotation)

a. Bush boasted of 'my [expletive] reading list'.

b. Every boy liked 'the gift [his $_{i}$ uncle's name] gave me'.

c. The politician admitted that she 'lied my way into [redacted]'.

(46) (Semantic unquotation)

a. Bush boasted of 'my [eclectic] reading list'.

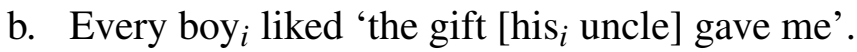

c. The politician admitted that she 'lied my way into [her job]'.

A syntactic unquote describes an expression, such as an expletive or a name, that can be plugged into a hole in a quoted expression. In contrast, a semantic unquote denotes a value, such as an uncle or a job, that can be plugged into the composition of a quoted meaning. In (46), Bush could have used neither the word eclectic nor eckullectic, each boy could have referred to his uncle using a different expression, and the politician could have said not my job but this despicable position of deception. The mixed quote in (47) exemplifies the ambiguity between syntactic and semantic unquotation: Bush could have said deafening inaudibly or in-fuckingaudible audibly.

(47) Bush complained about the 'utterly [inaudible] loudspeakers' in the room.

\subsection{The Prevalence of Unquotation}

In general, unquotation is a strategy for quoting a construction without quoting a complete constituent that it occurs in. Although the register of writing square brackets for unquotation is quite restricted, other instances of unquotation abound in speech as well as writing. I give three examples below, before implementing unquotation formally in the fragment.

First, non-constituents can be mixed-quoted, as Abbott (2003) showed with the following examples.

(48) David said that he had donated 'largish sums, to several benign institutions'.

Mary allowed as how her dog ate 'odd things, when left to his own devices'.

We can analyze these examples by postulating semantic unquotes at the edge of constituent mixed-quotes, as in (46c) above:

(50) David said that he had '[donated] largish sums, to several benign institutions'.

(51) Mary allowed as how her dog '[ate] odd things, when left to his own devices'.

By unquoting donated and ate, we correctly predict that, for example, (49) could be true even if all Mary said was (52).

(52) Fido devoured odd things, when left to his own devices. 
Furthermore, this analysis captures the fact that the mixed quotes in (48) and (49) include not just largish sums, to several benign institutions, odd things, and when left to his own devices, but also David's and Mary's juxtaposing these phrases after a transitive verb. That is why (49) could not be true if all Mary said was (53).

(53) Whereas under human supervision Fido already ate odd things, when left to his own devices he would even eat soap.

This subtlety is overlooked by Maier's (2008) analysis of breaking the quoted nonconstituent into two phrases.

Second, a register of informal banter is perfectly compatible with both syntactic and semantic unquotation, as long as it is practical to punctuate or intone the quotes to convey the speaker's intention. It is easier when the quoted language is distinct from the quoting language:

(54) John tried to show off his French at the restaurant the other day-he ordered 'à la mode [(the name of) some dessert] pour moi'.

To make fun of John, the quote includes his uttering à la mode before the dessert name.

Finally, just as a metavariable can occur inside Quine corners, a syntactic unquote can occur inside a pure quote:

(55) Ralph scribbled 'I suspect that [Ortcutt's beach alias] is a spy' on a notepad.

(56) 'I suspect that [Ortcutt's beach alias] is a spy' has ten words.

As one might expect, semantic unquotation cannot occur inside a pure quote unless it is clear from context how to reify the unquoted value into a unique expression in the quoted language that means it.

(57) ? Ralph scribbled 'I suspect that [Ortcutt] is a spy' on a notepad.

(58) * 'I suspect that [Ortcutt] is a spy' has ten words.

The hearer of (57) may be able to guess what expression Ralph chose to use to mean Ortcutt, but it is impossible to do so for (58).

\subsection{Rules for Unquotation}

We add the two rules below to our fragment, for syntactic and semantic unquotation respectively.

$$
\begin{array}{ll}
A^{\prime}::=[\mathrm{DP}] & \llbracket A^{\prime} \rrbracket(w)(i)=\llbracket \llbracket \mathrm{DP} \rrbracket(w) \rrbracket_{w}^{i} \\
A^{\prime}::=[A] & \llbracket A^{\prime} \rrbracket(w)(i)=\llbracket A \rrbracket
\end{array}
$$

Both kinds of unquotation turn an unprimed category into a primed category, so they can only occur inside quotation. (Primed categories and unquotation rules are thus analogous to slash categories and gap rules.) 
The rule (59) for syntactic unquotation applies only to a DP that denotes an expression, such as his uncle's name in (45b). We let such a syntactic unquote simply denote the character of that expression $\llbracket \mathrm{DP} \rrbracket(w)$. Strictly speaking, this rule does not handle expletive in (45a) and redacted in (45c), because they are not DPs. We regard these commonplace syntactic unquotes as abbreviations for an expletive and $a$ redacted expression.

The rule (60) turns any expression in our language into a semantic unquote. We let a semantic unquote denote a character that ignores its context argument $i$. The unquoted content $\llbracket A \rrbracket$ is evaluated not at the world $w$ where the unquote is evaluated, but at the world where the containing quote is eventually evaluated. In other words, this semantic rule gives rise to de-dicto readings. For example, the reading of (61) that (60) gives rise to is roughly equivalent to interpreting the spy de dicto in (62). That is, in each of Ralph's desired worlds, he finds the spy in that world, not necessarily the spy in the actual world.

(61) Gripped by paranoia, Ralph 'wanted to find [the spy] and shoot him myself'.

(62) Gripped by paranoia, Ralph wanted to find the spy and shoot him himself.

It is trivial to add another version of (60) that gives rise to de-re readings: just set $\llbracket A^{\prime} \rrbracket(w)(i)\left(w^{\prime}\right)$ to $\llbracket A \rrbracket(w)$ instead of $\llbracket A \rrbracket\left(w^{\prime}\right)$, so that the character denoted by the semantic unquote is not only context-insensitive but also rigid. However, in a larger and more realistic grammar, this additional rule may well be obviated by another means of generating de-re readings, such as quantifying in.

I have not shown in this fragment how to actually generate any quantifiers, such as an expletive, a redacted expression, and every boy in (45b) and (46b), or any bound pronouns, as in his uncle. Quantification and binding can be dealt with by generalizing the type-lifting that is already woven throughout the fragment to deal with intensionality (Shan 2001). The grammar then juggles the multiple levels of type-lifting (Barker and Shan 2008) that arise in the quoting and quoted languages.

\section{Beyond Mixed Quotation}

So far, I have given a formal account of mixed quotation. The fragment enforces well-formedness conditions and supports both kinds of unquotation, by incorporating syntactic distinctions and combination modes from quoted languages. The semantics generalizes causal reference to individuals and kinds, by modeling what quoted speakers mean and hence their mental entities.

In the rest of this paper, I take a closer look at the representation of utterance contexts, so as to show that this account of mixed quotation applies more broadly to pure quotation and the rest of language.

What is in an utterance context? In the fragment above, the semantic rules provide only one way to introduce contexts, namely by quotation (in (32)). There is also only a limited number of ways to use contexts, namely by quoted combination modes (in (27), (28), (41), and (42)) and by syntactic unquotation (in (59)). Given 
that these are the only ways to introduce and use contexts, we can regard a context as simply a function from (a possible world and) a quoted expression or combination mode. Formally, this shift in perspective is merely a matter of replacing the quoted context 'Bush English' in (32) by the function $\lambda w . \lambda e . \llbracket e \rrbracket_{w}^{B u s h ~ E n g l i s h ~ a n d ~ r e p l a c i n g ~}$ $\llbracket \cdot \rrbracket_{w}^{i}$ everywhere else by $i(w)(\cdot)$. If we only care about a few quoted expressions and combination modes, we can even regard the function $i(w)$ concretely as a small lookup table, such as a quadruple

$$
\left(\llbracket \text { eckullectic } \rrbracket_{w}^{i}, \llbracket \text { reading list } \rrbracket_{w}^{i}, \llbracket A::=A / B B \rrbracket_{w}^{i}, \llbracket A::=B B \backslash A \rrbracket_{w}^{i}\right) .
$$

\subsection{Pure Quotation}

Although the shift above does not change any of the fragment's predictions, it dissociates our notion of quotable contexts from any metaphysical notion of utterance contexts. Our interpretation function $i(w)$ only has to be some mathematical function whose domain includes quoted expressions and combination modes. In particular, we can recover pure quotation by letting $i(w)$ be the identity function, ${ }^{4}$ essentially a Herbrand interpretation. We just add the following rule:

$$
A::={ }^{\prime} A^{\prime}, \quad \llbracket A \rrbracket(w)=\llbracket A^{\prime} \rrbracket(w)(\lambda w . \lambda e . e)
$$

This new rule straightforwardly generates the pure and direct quotes in (3) and (4). It also works with the rule for syntactic unquotation in (59) to generate sentences such as (55) and (56). It is incompatible with the rule for semantic unquotation in (60), so the bad examples (57) and (58) are ruled out.

Pure quotation is one extreme on a spectrum of hyperintensionality. At the other end is indirect quotation, which some would say is no quotation at all. We can recover indirect quotation using another interpretation function, $\lambda w . \lambda e . \llbracket e \rrbracket$, which appeals to the semantics of our own language. Mixed quotation sits somewhere in the middle of the spectrum.

\subsection{The Rest of Language}

I have suggested previously that mixed quotation subsumes all of language except coinage (Shan 2007): Each word or construction we use was acquired from, and can be analyzed as a mixed quote of, some utterance (perhaps a generic one). For example, the ordinary sentence Alice saw Bob can be cobbled together from mixed quotes of Alice on one utterance occasion, Bob on another, and saw on a third, combined using semantic unquotation as shown below.

\section{'['Alice'] saw ['Bob']'}

\footnotetext{
${ }^{4}$ That is, the identity function on quoted expressions and combination modes. A combination mode, such as $A::=B B \backslash A$, is here a function that maps an ordered pair of subexpressions to their combined expression.
} 
In fact, the quoted utterances of Alice, Bob, and saw are probably not coinages but mixed quotes themselves. If so, the following notation would be more accurate.

$$
\text { "[["Alice"]] saw [["Bob"]]" }
$$

This subsumption underscores the expressivity of mixed quotation for curating meanings from other minds, including their curation from yet other minds. However, these nested mixed quotes are so complex in syntax and semantics as to appear psychologically implausible. Surely, when we say merely Alice saw Bob in the usual way, we do not reason about what others use saw to mean that they think yet others use saw to mean.

It turns out that we can competently speak nested mixed quotes as in (66) without performing nested mental reasoning all the time. As soon as we learn what saw is used to mean-in other words, as soon as we shrink our set of belief worlds $w$ enough that $\llbracket$ saw $\rrbracket_{w}^{i}$ is constant across them-the mixed quote 'saw' becomes equivalent to an ordinary word saw whose semantic content, involving no diagonalization, is that constant, which we might as well call «saw》. This equivalence is same as the one between 'eckullectic' and eclectic described in (35).

In general, each mixed-quoted word or construction whose syntax and semantics we know can be merged into our own unquoted language, the extra level of quotation ignored thereafter. To learn a language, be it French or Bush English, is thus to move its words and constructions along the spectrum of hyperintensionality from mixed quotation to indirect quotation, or no quotation at all.

\section{References}

Abbott, Barbara: 2003, 'Some Notes on Quotation', In de Brabanter (2003), 13-26. Barker, Chris: 2007, 'Direct Compositionality on Demand', In Barker and Jacobson (2007), 102-131, Oxford University Press.

Barker, Chris and Pauline Jacobson (eds.): 2007, 'Direct Compositionality'. New York: Oxford University Press.

Barker, Chris and Chung-chieh Shan: 2008, 'Donkey Anaphora is In-Scope Binding', Semantics and Pragmatics 1, 1-46.

Bawden, Alan: 1999, 'Quasiquotation in Lisp', in O. Danvy (ed.), Proceedings of the 1999 ACM SIGPLAN Workshop on Partial Evaluation and SemanticsBased Program Manipulation, 4-12. BRICS.

de Brabanter, Philippe (ed.): 2003, Hybrid Quotations (Belgian Journal of Linguistics 17(1)). John Benjamins, Amsterdam.

Cappelen, Herman and Ernie Lepore: 2003, 'Varieties of Quotation Revisited', In de Brabanter (2003), 51-75.

Davidson, Donald: 1979, 'Quotation', Theory and Decision 11, 27-40.

Geurts, Bart and Emar Maier: 2003, 'Quotation in Context', In de Brabanter (2003), 109-128.

Kaplan, David: 1989, 'Demonstratives: An Essay on the Semantics, Logic, Metaphysics, and Epistemology of Demonstratives and Other Indexicals', in J. 
Almog, J. Perry, and H. Wettstein (eds.), Themes from Kaplan, 481-563. Oxford University Press, New York.

Kripke, Saul A.: 1980, Naming and Necessity. Harvard University Press, Cambridge.

Maier, Emar: 2008, 'Breaking Quotations', in K. Satoh, A. Inokuchi, K. Nagao, and T. Kawamura (eds.), New Frontiers in Artificial Intelligence: Revised Selected Papers from JSAI 2007 Conference and Workshops, 187-200. Springer, Berlin.

Potts, Christopher: 2003, The Logic of Conventional Implicatures, Doctoral Dissertation, University of California, Santa Cruz.

Potts, Christopher: 2007, 'The Dimensions of Quotation', In Barker and Jacobson (2007), 405-431, Oxford University Press.

Putnam, Hilary: 1975, 'The Meaning of "Meaning"', in K. Gunderson (ed.), Language, Mind and Knowledge, No. 7 in Minnesota Studies in the Philosophy of Science, 131-193. University of Minnesota Press, Minneapolis.

Quine, Willard Van Orman: 1940, Mathematical Logic. Harvard University Press, Cambridge.

Shan, Chung-chieh: 2001, 'Monads for Natural Language Semantics', in K. Striegnitz (ed.), Proceedings of the ESSLLI-2001 Student Session, 285-298. 13th European Summer School in Logic, Language and Information, Helsinki.

Shan, Chung-chieh: 2007, 'Causal Reference and Inverse Scope as Mixed Quotation', in M. Aloni, P. Dekker, and F. Roelofsen (eds.), Proceedings of the 16th Amsterdam Colloquium, 199-204. Institute for Logic, Language and Computation, Universiteit van Amsterdam.

Stalnaker, Robert C.: 1978, 'Assertion', in P. Cole (ed.), Pragmatics, No. 9 in Syntax and Semantics, 315-332. Academic Press, San Diego, CA. 